\title{
Ablative Endocrine Surgery
}

National Cancer Institute

\section{Source}

National Cancer Institute. Ablative Endocrine Surgery. NCI Thesaurus. Code C15467.

Destructive or removal of any part of the endocrine system by surgical means, including the use of lasers or electrical current. 